\title{
Human papillomavirus and vaccination: knowledge, attitudes, and behavioural intention in adolescents and young women in Italy
}

\author{
G Di Giuseppe', R Abbate', G Liguori', L Albano' and IF Angelillo*,I \\ 'Department of Public, Clinical and Preventive Medicine, Second University of Naples, Naples, Italy; 'Chair of Hygiene, University of Naples 'Parthenope', \\ Naples, Italy
}

\begin{abstract}
This study assesses knowledge, attitudes, and behavioural intention towards human papillomavirus (HPV) infection and vaccination in a random sample of 1348 adolescents and young women aged 14-24 years in Italy. A self-administered anonymous questionnaire covered demographics; knowledge about HPV infection, cervical cancer, and HPV vaccine; the perceived risk for contracting HPV infection and/or for developing cervical cancer, the perceived benefits of a vaccination to prevent cervical cancer, and willingness to receive an HPV vaccine. Only 23.3\% have heard that HPV is an infection of the genital mucosa and about cervical cancer. Those older, with at least one parent who is a health care professional, with personal, familiar, or friendly history of cervical cancer, and having underwent a health checkup in the last year with information about HPV vaccination were significantly more knowledgeable. Risk perception scores (range: I - 10) of contracting HPV infection and of developing cervical cancer were 5.8 and 6.5. Older age, not having a parent who is a health care professional, having had a personal, familiar, or friendly history of cervical cancer, and need of additional information were predictors of the perceived susceptibility of developing cervical cancer. The vast majority professed intent to receive an HPV vaccine and the significant predictors were having at least one parent who is a health care professional, a high perceived risk of contracting HPV infection and of developing cervical cancer, and a high belief towards the utility of a vaccination for preventing cervical cancer. Knowledge about HPV infection and cervical cancer should be improved with more attention to the benefit of HPV vaccination.

British Journal of Cancer (2008) 99, 225 -229. doi: I 0. I038/sj.bjc.6604454 www.bjcancer.com

(c) 2008 Cancer Research UK
\end{abstract}

Keywords: attitudes; behavioural intention; cervical cancer; human papillomavirus; Italy

Human papillomavirus (HPV) is one of the most common sexually transmitted infections in sexually active adolescent girls and young women of several economically developed countries (Richardson et al, 2003; Syrjänen et al, 2005; Kitchener et al, 2006; Dunne et al, 2007). Strong evidence has been observed for the role of persistent high-risk HPV types (16 and 18), in the aetiology of cervical cancer, as worldwide they are responsible for approximately $70 \%$ of all cases (Clifford et al, 2006; Markowitz et al, 2007). Cervical cancer is the second most common cancer among women worldwide, with half a million who develop it annually and more than half of the cases die as a result (Parkin et al, 2005; Cutts et al, 2007).

Highly immunogenic and safe HPV vaccines recently have been licensed for use, the first explicitly designed to prevent cancer induced by a virus, which can reduce the morbidity and mortality of cervical cancer by offering protection to HPV types 16 and 18 . Because the vaccine has the maximum benefit when given before a person becomes sexually active, the Advisory Committee on Immunization Practices of the Centers for Disease Control and

\footnotetext{
* Correspondence: Professor IF Angelillo, Department of Public, Clinical and Preventive Medicine, Second University of Naples, via Luciano Armanni 5, 80I38 Naples, Italy; E-mail: italof.angelillo@unina2.it Preliminary results were presented at the $X$ National Conference of the Italian Public Health Association, October 14-16, 2007, Pisa, Italy. Revised 9 May 2008; accepted 9 May 2008
}

Prevention (CDC) and the American Academy of Pediatrics, have recommended routine vaccination for girls aged 11 or 12 years (Clifford et al, 2006; American Academy of Pediatrics, 2007). In Italy, the Ministry of Health has recently approved a national programme for free HPV vaccination for girls aged 12 (Ministero della Salute, 2007).

Widespread acceptance of HPV vaccine is likely to provide enormous public health benefits (Garnett et al, 2006; Bosch et al, 2008; Goldhaber-Fiebert et al, 2008) and it must be administered before initiating sexual activity (Stanley et al, 2006; Markowitz et al, 2007; Saslow et al, 2007) and hence would be most effective if offered during early adolescence (Adams et al, 2007; Cutts et al, 2007). Because most sexually active individuals will become exposed to the virus throughout their lives and because the HPV vaccine is available, it is extremely important to gather data on HPV infection and vaccination. Such questioning has already taken place in several studies which mainly address vaccine acceptability among parents (Olshen et al, 2005; Brabin et al, 2006, 2007; Dempsey et al, 2006; Dinh et al, 2007; Marlow et al, 2007) and health care workers (Kahn et al, 2003, 2005; Aldrich et al, 2005; Riedesel et al, 2005; Moreira et al, 2006a, b; Duval et al, 2007; Hopenhayn et al, 2007; Waller et al, 2007; Woodhall et al, 2007), but very little research has targeted adolescents. Therefore, this study attempts to generate information about knowledge, attitudes, and behavioural intention towards HPV infection and vaccination among adolescents and young women in Italy. 


\section{MATERIALS AND METHODS}

This cross-sectional investigation was conducted from March through May 2007 and the target population comprised 1348 women aged 14-24 years. The sample was selected with a twostage cluster method. Seven faculties that cover a wide range of disciplines (economics, education, law, literature, and sport sciences) in two Universities and six public secondary schools in the geographic area of the Campania region, in the South of Italy, were randomly chosen. A simple random technique was adopted in selecting students from each faculty and school.

Before the study, a meeting with the Head and the Dean of each randomly selected school and faculty was arranged to present the project and the permission and collaboration were obtained. A questionnaire was pilot tested on 25 students before initiation of the survey, the objective of which was to identify the basic questions most valid for testing the comprehensiveness and relevance of our terms. As part of the process of informed consent, all potential candidates were invited to participate through a letter containing information about purpose and objectives, also indicating that participation in the survey was voluntary, and that privacy and confidentiality would be strictly protected as no personal identifiers were included in the questionnaire and data would be presented only in an aggregated manner. For those under 18 years of age, a separate information letter was addressed to the parents. These policies were also printed explicitly on the front page of the questionnaire. Provided that respondent and parental consent were obtained, data were collected by self-administered anonymous structured questionnaire administered with a reply envelope. The questionnaires were delivered to students in their classroom one time and were completed at the time of distribution. A respondent's consent was taken into account while filling the questionnaire. The questionnaire was used to explore several topics, including: (1) student characteristics, included age, marital status, employment status, whether they were living alone or not, and whether they have had a personal, familiar, or friendly history of cervical cancer; (2) knowledge about HPV infection (definition and modes of transmission), cervical cancer (definition and preventive measures), and availability of an HPV vaccine; (3) attitudes pertaining to assess whether or not the respondent perceived a risk for contracting HPV infection and/or for developing cervical cancer, opinions about the perceived benefits of a vaccination to prevent cervical cancer, and their willingness to receive an HPV vaccine; (4) behaviour, included questions whether or not respondents have ever had sex, age at first sex, number of acts of intercourse and of partners in the last year, and how frequently they were protected by condoms during intercourse with the partner(s) in the last year; health-promoting behaviour was assessed with questions about how often the respondent underwent a health checkup in the last year, whether respondents' health care provider informed them about HPV vaccine, and whether they have received the HPV vaccine; (5) sources of and needs of information regarding HPV vaccine and cervical cancer.

For items regarding their knowledge, participants were asked to provide answers with options for 'no', 'do not know', and 'yes'. All attitudes were measured on a 10-point Likert scale with a score ranging from 1 to 10 . The responses for the two questions about perceived risk were recorded as 1 for no risk at all and 10 for very high perceived risk; for the perceived benefits of a vaccination to prevent cervical cancer, 1 for not at all and 10 for high utility; and for their willingness to receive HPV vaccine, 1 for unwillingness and 10 for willingness. The respondents were also asked to report directly reason(s) for willingness or unwillingness to receive HPV vaccine. Questions pertaining to behaviours were close ended with nominal or categorical (yes or no) responses; the frequency of the use of condoms in the last year was measured on a five-point Likert scale ranging from 'never' to 'every time'. Ethics committee approval was obtained.

\section{Statistical analysis}

We analysed multivariable logistic and linear regression models using a forward stepwise procedure to assess the independent predictors of the following outcomes of interest: have heard about HPV infection and cervical cancer (Model 1); perception of risk of developing cervical cancer (Model 2); willingness to receive an HPV vaccine (Model 3). The following explanatory variables were included in all models: age (continuous), at least one parent is a health care professional $(0=$ no, $1=$ yes $)$, personal, familiar, or friendly history of cervical cancer $(0=$ no, $1=$ yes $)$, at least a health checkup in the last year and the physician informed them about HPV vaccination $(0=$ no, $1=$ yes $)$, number of sexual partners in the last year $(0=0,1=1,2=\geqslant 2)$, and need of information about HPV vaccination and cervical cancer $(0=$ no, $1=$ yes $)$. The following variables were also included: have already heard about HPV infection and cervical cancer $(0=$ no, $1=$ yes $)$ in Models 2 and 3; perception of risk of contracting HPV infection (continuous), perception of risk of developing cervical cancer (continuous), and perceived benefits of a vaccination to prevent cervical cancer (continuous) in Model 3. The significance level for variables entering the logistic and linear regression models was set at 0.2 and for removing at 0.4 . Odds ratios (ORs) with $95 \%$ confidence intervals (CIs) were used in the logistic regression analyses. All reported $P$-values were assessed using two-sided tests and statistical significance was taken as a cutoff of $P \leqslant 0.05$ throughout. The statistical package Stata (Version 8.1) was used for the analysis (Stata Corporation, 2003).

\section{RESULTS}

Subjects who participated in the survey were 1341 and a response rate of $99.5 \%$ was achieved. The mean age of participants was 19 years, almost all were single, the majority was full-time students, $8.9 \%$ had at least one parent who is a health care professional, and $16.3 \%$ has had a personal, familiar, or friendly history of cervical cancer.

The distribution of respondents who answered correctly to each of the different items of the knowledge about HPV infection and cervical cancer are presented in Table 1. Less than one-third $(29.8 \%)$ reported that they have heard that HPV is one of the most common infections of the genital mucosa and three-quarters of them identified that the infection is primarily transmitted through sexual intercourse. Only half reported having heard of cervical cancer before and 60.2 and $34.8 \%$ of them, respectively, know that the majority of cases and deaths can be prevented through detection of precancerous changes in the cervix by using the Pap test and that condom use reduce the risk for HPV and cervical cancer. Less than half $(42.1 \%)$ knows that the vaccine was a preventive measure, but only $15.3 \%$ knows that a vaccine is available in Italy. Overall, only $23.3 \%$ have heard that HPV is one of the most common infections of the genital mucosa and about cervical cancer. The adjusted ORs for the likelihood of having heard about HPV infection and cervical cancer by several variables are presented in Table 2. After multivariate logistic adjustments, this model reveals that older age was significantly associated with the outcome as the adjusted OR was 1.14 (95\% CI $1.08-1.2)$ for every 1-year increment. This model also reveals that female subjects having at least one parent who is a health care professional (OR $=1.66$; 95\% CI 1.07-2.58), having had a personal, familiar, or friendly history of cervical cancer $(\mathrm{OR}=3.04 ; 95 \% \mathrm{CI}$ 2.26-5.11), and having underwent a health checkup in the last year and the physician informed them about HPV vaccination $(\mathrm{OR}=2.44 ; 95 \%$ CI $1.23-4.85)$ were associated with having higher odds of having heard about HPV vaccination compared to those who have not (Model 1 in Table 2).

Risk perception of contracting HPV infection and of developing cervical cancer was similar with the respondents inferring a 
level of perception with scores of 5.8 and 6.5, respectively and only 12.7 and $19.9 \%$ perceived themselves at a high risk, respectively by responding ' 10 '. Table 2 presents the adjusted mean of perceived risk from a multiple linear regression model that controlled for the contribution of several variables. Older age, not having at least one parent who is a health care professional, having had a personal, familiar, or friendly history of cervical cancer, and need of additional information about this cancer and HPV vaccination were significantly the predictors of the perceived susceptibility of

Table I Knowledge about HPV and cervical cancer of the study population

\begin{tabular}{lcc}
\hline & N & $\%$ \\
\hline Have heard about HPV infection & 399 & 29.8 \\
Have heard about cervical cancer & 706 & 52.6 \\
& \multicolumn{2}{c}{ Correct response } \\
\cline { 2 - 3 } & $\mathbf{N}$ & $\%$ \\
\hline Modes of transmission of HPV & & \\
Complete sexual intercourse (true) & 300 & 75.2 \\
Incomplete sexual intercourse (true) & 116 & 29.1 \\
Needle sharing (false) & 91 & 22.9 \\
Pregnancy (false) & 70 & 17.5 \\
Vaginal delivery (true) & 63 & 15.8 \\
& & \\
Preventive measures for cervical cancer & & 60.2 \\
Pap test (true) & 425 & 42.4 \\
Late start of sexual activity (true) & 299 & 42.1 \\
HPV vaccination (true) & 297 & 36.3 \\
Oral contraceptive (false) & 256 & 34.8 \\
Condom use (true) & 246 & \\
\hline
\end{tabular}

$\mathrm{HPV}=$ human papillomavirus. ${ }^{\mathrm{a}}$ Only for those who reported that they have heard about HPV infection. ${ }^{b}$ Only for those who reported that they have heard about cervical cancer. developing cervical cancer (Model 2 in Table 2). Subjects were asked about their belief towards the utility of an HPV vaccination for preventing cervical cancer and whether they would consider it in future, with a mean total score of 8.7 and 7.7, respectively. The vast majority (81.7\%) professed intent to obtain an HPV vaccine in future. Multiple logistic regression was used to examine the probability of willingness to receive the HPV vaccination and the adjusted ORs are presented in Table 2 . The significant predictors of the willingness to receive this vaccine were high perceived risk of contracting HPV infection (OR $=1.18 ; 95 \%$ CI $1.08-1.29)$ and of developing cervical cancer (OR $=1.09 ; 95 \%$ CI $1.01-1.18$ ), a high belief in the utility of a vaccination for preventing cervical cancer $(\mathrm{OR}=1.33 ; 95 \% \mathrm{CI} 1.24-1.42)$, and having one parent who is a health care professional (OR $=1.86$; 95\% CI 1.03-3.39; Model 3 in Table 2).

For those who stated that they would consider the HPV vaccination in future, the main reasons were the belief that the vaccination reduces the risk of contracting HPV infection $(73.8 \%)$ and of developing cervical cancer $(59.7 \%)$, and felling at risk $(10 \%)$. By contrast, for those who stated that they would not consider HPV vaccine, the most common reasons were the belief that the vaccine was dangerous (59.6\%) and that they feel to be not at risk $(42 \%)$.

According to sexual behaviour pattern, $45.4 \%$ reported current or previous sexual activity, and the mean age of first sexual intercourse of the sexually experienced was 17 years. Overall, only $27.1 \%$ of those sexually experienced reported that they have always used a condom during the intercourse in the last year. Approximately, half had at least one health checkup over the last year $(48 \%)$ and for only $3 \%$, the physician informed them about HPV vaccination.

When asked about their main source of information of HPV infection and cervical cancer, only $6 \%$ claimed health professionals. Among those who knew that a vaccine is available in Italy, 62.7 and $17.4 \%$ had received such information from television/ mass media and from health professionals, respectively. Almost all respondents wanted more information about HPV vaccination (94.4\%) and cervical cancer (95.7\%).

Table 2 Logistic and linear regression models' results

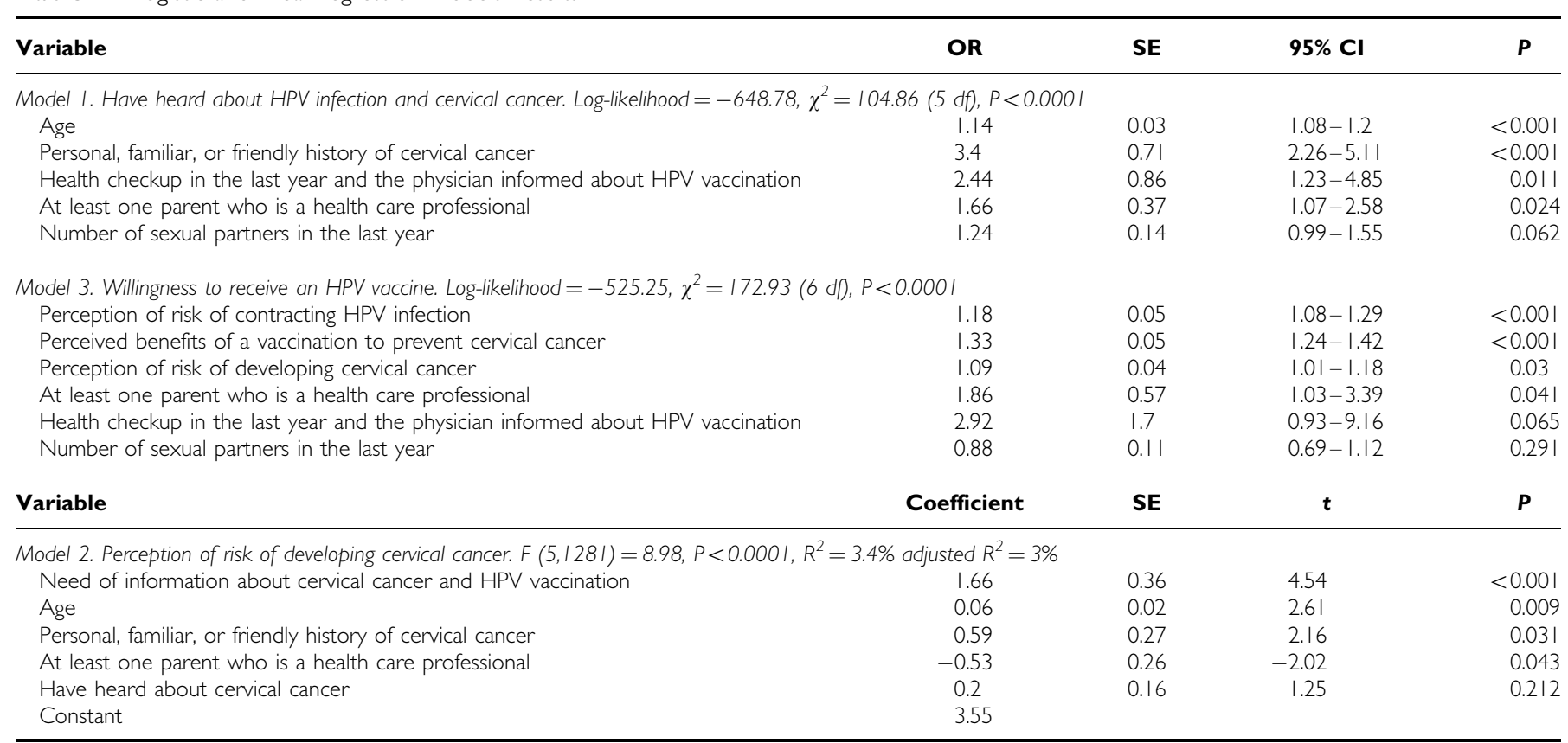

$\mathrm{Cl}=$ confidence interval; $\mathrm{HPV}=$ human papillomavirus; $\mathrm{OR}=$ Odds ratio; $\mathrm{SE}=$ standard error. 


\section{DISCUSSION}

This large study expands the previous reports and findings on knowledge, attitudes, and behaviour towards HPV infection, vaccination, and cervical cancer patterns among young women are of particular interest, because the responses reflect real issues in this population setting. This study included only women, mainly because when it has been conducted, the available HPV vaccine was approved for use in female subjects only and, as already indicated, international guidelines recommend vaccination of girls aged 11-12 years and in our country, it was free of charge only for girls of 12 years of age. Moreover, this approach is supported by cost-effectiveness analyses that have indicated that the additional vaccination of boys has a limited effect compared to girls-only vaccination (Taira et al, 2004; Newall et al, 2007).

Our results indicate that women's knowledge about HPV infection and cervical cancer was remarkably poor, as only $23.3 \%$ ever heard of them; particularly striking was that a very high proportion did not know that vaccination can prevent this cancer and that they were unaware of such a vaccine in Italy. This finding was lower than those observed in earlier surveys. Indeed, $33 \%$ of 397 adolescents, mean age of 15 years in Finland, have heard of HPV before addressing the questionnaire (Woodhall et al, 2007 ) and $58 \%$ of 811 university students in the United Kingdom have heard of HPV (Waller et al, 2007). Moreover, our prevalence that $75.2 \%$ knew about sexual transmission of HPV is higher than the $66.7 \%$ found in a study of 204 women aged 16-23 years attending a public outpatient gynaecological clinic in Brazil (Moreira et al, 2006a). As in these prior studies, our results suggest that the continued efforts are urgently needed to educate young women. As predicted, the concept of physician influence is clearly important in this context of educating and counselling patients and potentially it is at this level of initial contact between physician and patient when interventions designed to increase knowledge about HPV and cervical cancer might be best implemented. Having a physician visit for a checkup and information about HPV vaccination was an important predictor of a higher level of knowledge and it is also not surprising that we observed an association, although not reaching statistical significance, between the willingness to receive the vaccination and information from a physician. Indeed, there is a group of respondents who consider HPV vaccination in future simply if their physician engaged them in a discussion about HPV vaccine. However, if patients remain uneducated about the opportunities, then logically compliance and adherence with recommended vaccination will stay forever low. So it is reasonable that an endorsement from a trusted physician would most likely result in acceptance of HPV vaccination now that guidelines are in place and the vaccination is more popular.

Our study highlights the positive attitude of the sample, as a vast majority $(81.7 \%)$ professed intent to receive the HPV vaccination. This finding is consistent with the already mentioned study in Finland where $86 \%$ declaring they would accept the vaccination (Woodhall et al, 2007); with a study in the United States, with $85 \%$ of participants 18-30 years of age, recruited from the community and clinical sites, who reported that they would be extremely or very likely to receive an HPV vaccine (Kahn et al, 2003). A lower value was reported in Brazil with $72 \%$ who would enroll in an HPV vaccine trial (Moreira et al, 2006a). Finally, in a population-based study in two Appalachian Kentucky counties, $92.1 \%$ of women aged 18-29 years indicated an interest in HPV vaccination (Hopenhayn et al, 2007).

Study participants showed that 12.7 and $19.9 \%$, respectively, perceived themselves likely to acquire the infection and the disease by responding ' 10 '. In the already mentioned studies of Brazil and United States, $68.1 \%$ perceived themselves at moderate-to-high risk (Moreira et al, 2006b) and 54\% strongly or somewhat agreed that they were at risk of developing cervical cancer (Kahn et al, 2003). Further, the results of the logistic regression model showed that women who perceived a high risk of contracting HPV infection were more likely to receive the vaccination in future. That finding of an association between willingness to receive the vaccination and self-perceived risk of contracting infection agrees with a prior study (Moreira et al, 2006a). Why persons at higher self-perceived risk are the most willing to obtain vaccination is clear. Perceptions of heightened risk do serve as an effective stimulus to pursue vaccination. Another notable finding was that among the respondents who stated that they would not consider HPV vaccination in future, their most commonly stated reason appeared to be the belief that the vaccine was dangerous. These findings have implications for practice in public health as physicians, mainly those in primary care, are obligated to evaluate and rectify, when appropriate, any misperceptions or confusions about vaccination.

This study has some potential limitations. First, the research questions were investigated by a cross-sectional study design. Such design precludes determination of causal relationships between different factors and outcomes. Second, information was gathered by self-reported questionnaire and so the possibility exists that some responses may have reported incorrect information. Notwithstanding this criticism, the anonymity of the study may have reduced the variation in the responses. This study, however, displays important strengths: first, it provides information from a large number of participants and this allows exploration of very weak associations between variables; second, the data were essentially complete; third, the participation rate was very high, probably reflecting heightened interest for this survey.

In conclusion, our results suggest that the continuing efforts should be directed at focusing attention towards the benefits of following HPV vaccination recommendations emphasising those factors positively associated. There is also an urgent need for designing educational interventions to improve the level of knowledge about HPV infection and cervical cancer.

\section{ACKNOWLEDGEMENTS}

This investigation was financed in part by a research grant of the Sanofi Pasteur MSD. This funding was obtained and administered through the Department of Public, Clinical and Preventive Medicine, Second University of Naples at Naples, Italy. We are grateful to all women who agreed to participate in the study.

\section{REFERENCES}

Adams M, Jasani B, Fiander A (2007) Human papilloma virus (HPV) prophylactic vaccination: challenges for public health and implications for screening. Vaccine 25: 3007-3013

Aldrich T, Becker D, García SG, Lara D (2005) Mexican physicians' knowledge and attitudes about the human papillomavirus and cervical cancer: a national survey. Sex Transm Infect 81: 135-141
American Academy of Pediatrics Committee on Infectious Diseases (2007) Prevention of human papillomavirus infection: provisional recommendations for immunization of girls and women with quadrivalent human papillomavirus vaccine. Pediatrics 120: 666-668

Bosch FX, Castellsagué X, de Sanjosé S (2008) HPV and cervical cancer: screening or vaccination? Br J Cancer 98: 15-21 
Brabin L, Roberts SA, Farzaneth F, Kitchener HC (2006) Future acceptance of adolescent human papillomavirus vaccination: a survey of parental attitudes. Vaccine 24: 3087-3094

Brabin L, Roberts SA, Kitchener HC (2007) A semi-qualitative study of attitudes to vaccinating adolescents against human papillomavirus without parental consent. BMC Public Health 7: 20

Clifford G, Franceschi S, Diaz M, Muñoz N, Villa LL (2006) Chapter 3: HPV type-distribution in women with and without cervical neoplastic diseases. Vaccine 24(Suppl 3): S26-S34

Cutts FT, Franceschi S, Goldie S, Castellsagué X, de Sanjose S, Garnett G, Edmunds WJ, Claeys P, Goldenthal KL, Harper DM, Markowitz L (2007) Human Papillomavirus and HPV vaccines: a review. Bull WHO 85: $719-726$

Dempsey AF, Zimet GD, Davis RL, Koutsky L (2006) Factors that are associated with parental acceptance of human papillomavirus vaccines: a randomized intervention study of written information about HPV. Pediatrics 117: 1486 - 1493

Dinh TA, Rosenthal SL, Doan ED, Trang T, Pham VH, Tran BD, Tran VD, Phan GA, Chu HK, Breitkopf CR (2007) Attitudes of mothers in Da Nang, Vietnam toward a human papillomavirus vaccine. J Adolesc Health 40: $559-563$

Dunne EF, Unger ER, Sternberg M, McQuillan G, Swan DC, Patel SS, Markowitz LE (2007) Prevalence of HPV infection among females in the United States. JAMA 297: 813-819

Duval B, Gilca V, McNeil S, Dobson S, Money D, Gemmill IM, Sauvageau C, Lavoie F, Ouakki M (2007) Vaccination against human papillomavirus: a baseline survey of Canadian clinicians' knowledge, attitudes and beliefs. Vaccine 25: 7841 - 7847

Garnett GP, Kim JJ, French K, Goldie SJ (2006) Modelling the impact of HPV vaccines on cervical cancer and screening programmes. Vaccine 24(Suppl 3): $178-186$

Goldhaber-Fiebert JD, Stout NK, Salomon JA, Kuntz KM, Goldie SJ (2008) Cost-effectiveness of cervical cancer screening with human papillomavirus DNA testing and HPV-16, 18 vaccination. J Natl Cancer Inst 100: $308-320$

Hopenhayn C, Christian A, Christian WJ, Schoenberg NE (2007) Human papillomavirus vaccine: knowledge and attitudes in two Appalachian Kentucky counties. Cancer Causes Control 18: 627-634

Kahn JA, Rosenthal SL, Hamann T, Bernstein DI (2003) Attitudes about human papillomavirus vaccine in young women. Int J STD AIDS 14: $300-306$

Kahn JA, Zimet GD, Bernstein DI, Riedesel JM, Lan D, Huang B, Rosenthal SL (2005) Pediatricians' intention to administer human papillomavirus vaccine: the role of practice characteristics, knowledge, and attitudes. J Adolesc Health 37: $502-510$

Kitchener HC, Almonte M, Wheeler P, Desai M, Gilham C, Bailey A, Sargent A, Peto J, ARTISTIC Trial Study Group (2006) HPV testing in routine cervical screening: cross sectional data from the ARTISTIC trial. $\mathrm{Br} J$ Cancer 95: 56-61

Markowitz LE, Dunne EF, Saraiya M, Lawson HW, Chesson H, Unger ER, Centers for Diseases Control and Prevention, Advisory Committee on Immunization Practices (2007) Quadrivalent human papillomavirus vaccine: recommendations of the Advisory Committee on Immunization Practices (ACIP). MMWR Recomm Rep 56(RR-2): 1-24
Marlow LA, Waller J, Wardle J (2007) Parental attitudes to pre-pubertal HPV vaccination. Vaccine 25: $1945-1952$

Ministero della Salute (2007) L'impegno per la prevenzione del tumore della cervice uterina. Entro febbraio disponibile in Italia il primo vaccino contro questa malattia ma resta fondamentale l'attività di screening con il pap-test. Vaccino gratuito per tutte le ragazze al compimento dei dodici anni Available at www.ministerosalute.it/resources/static/primopiano/ 377/vaccino HPV.pdf (Accessed 5 February 2007)

Moreira Jr ED, de Oliveira BG, Silva Neves RC, Costa S, Karic G, Costa Filho JO (2006a) Assessment of knowledge and attitudes of young uninsured women toward human papillomavirus vaccination and clinical trials. J Pediatr Adolesc Gynecol 19: 81 - 87

Moreira ED, Oliveira BG, Ferraz FM, Costa S, Costa Filho JO, Karic G (2006b) Knowledge and attitudes about human papillomavirus, Pap smears, and cervical cancer among young women in Brazil: implications for health education and prevention. Int J Gynecol Cancer 16: 599-603

Newall AT, Beutels P, Wood JG, Edmunds WJ, Macintyre CR (2007) Costeffectiveness analyses of human papillomavirus vaccination. Lancet Infect Dis 7: 289-296

Olshen E, Woods ER, Austin SB, Luskin M, Bauchner H (2005) Parental acceptance of the human papillomavirus vaccine. I Adolesc Health 37: 248 - 251

Parkin DM, Bray F, Ferlay J, Pisani P (2005) Global Cancer Statistics, 2002. CA Cancer J Clin 55: 74-108

Richardson H, Kelsall G, Tellier P, Voyer H, Abrahamowicz M, Ferenczy A, Coutlée F, Franco EL (2003) The natural history of type-specific human papillomavirus infections in female university students. Cancer Epidemiol Biomarkers Prev 12: $485-490$

Riedesel JM, Rosenthal SL, Zimet GD, Bernstein DI, Huang B, Lan D, Kahn JA (2005) Attitudes about human papillomavirus vaccine among family physicians. I Pediatr Adolesc Gynecol 18: 391 - 398

Saslow D, Castle PE, Cox JT, Davey DD, Einstein MH, Ferris DG, Goldie SJ, Harper DM, Kinney W, Moscicki A, Noller KL, Wheeler CM, Ades T, Andrews KS, Doroshenk MK, Kahn KG, Schmidt C, Shafey O, Smith RA, Partridge EE, Garcia F (2007) American Cancer Society Guideline for Human Papillomavirus (HPV) vaccine use to prevent cervical cancer and its precursors. Ca Cancer J Clin 57: 7-28

Stanley M, Lowy DR, Frazer I (2006) Prophylactic HPV vaccines: underlying mechanism. Vaccine 24(Suppl 3): 106-113

Stata Corporation. Stata Reference Manual Release 8.1. College Station, TX, USA, 2003

Syrjänen S, Shabalova I, Petrovichev N, Podistov J, Ivanchenko O, Zakharenko S, Nerovjna R, Kljukina L, Branovskaja M, Juschenko A, Tosi P, Syrjänen K, NIS Cohort Study Group (2005) Age-specific incidence and clearance of high-risk human papillomavirus infections in women in the former Soviet Union. Int J STD AIDS 16: 217-223

Taira AV, Neukermans CP, Sanders GD (2004) Evaluating human papillomavirus vaccination programs. Emerg Infect Dis 10: 1915-1923

Waller J, Marlow L, Wardle J (2007) The association between knowledge of HPV and feelings of stigma, shame and anxiety. Sex Transm Infect 83: $155-159$

Woodhall SC, Lehtinen M, Verho T, Huhtala H, Hokkanen M, Kosunen E (2007) Anticipated acceptance of HPV vaccination at the baseline of implementation: a survey of parental and adolescent knowledge and attitudes in Finland. J Adolesc Health 40: 466-469 